\title{
Des Miss à part. Les concours de beauté au prisme des différences en Amérique latine
}

Grégory Deshoullière et Magda Helena Dziubinska

\section{OpenEdition}

1 Journals

Édition électronique

URL : https://journals.openedition.org/jsa/14915

DOI : 10.4000/jsa. 14915

ISSN : 1957-7842

Éditeur

Société des américanistes

Édition imprimée

Date de publication : 15 juin 2017

Pagination : 13-26

ISSN : 0037-9174

Référence électronique

Grégory Deshoullière et Magda Helena Dziubinska, « Des Miss à part. Les concours de beauté au prisme des différences en Amérique latine ", Journal de la Société des américanistes [En ligne], 103-1 | 2017, mis en ligne le 15 juin 2017, consulté le 02 septembre 2022. URL : http://

journals.openedition.org/jsa/14915; DOI : https://doi.org/10.4000/jsa.14915 


\title{
Des Miss à part. Les concours de beauté au prisme des différences en Amérique latine
}

\author{
Grégory Deshoullière et Magda Helena DziubinsKa *
}

Les concours de beauté sont devenus aujourd'hui un élément central des festivités dont les Amérindiens sont les principaux acteurs - les anniversaires des communautés natives au Pérou, le Jour des nationalités en Équateur, les fêtes patronales au Guatemala ou le Jour de l'Indien au Brésil, pour n'en nommer que quelques-unes ${ }^{1}$. Pourtant, les concours de beauté en Amérique latine ont pendant longtemps été l'apanage des sociétés nationales, certaines en ayant même fait une industrie d'État ${ }^{2}$. La littérature régionale a en effet mis en lumière le lien entre la célébration de la nation et la promotion d'un idéal féminin issu des élites urbaines ${ }^{3}$. L'histoire de ces concours au sein des États-nations d'Amérique latine reste néanmoins mal connue. L'hypothèse la plus souvent émise fait remonter leur diffusion à partir des États-Unis aux

* G. Deshoullière, EHESS/Laboratoire d'anthropologie sociale, 52, rue du Cardinal Lemoine, 75005 Paris [g.deshoulliere@inventati.org]; M. H. Dziubinska, LESC/EREA, 21, allée de 1'Université, 92023 Nanterre CEDEX [dziubinska@gmail.com].

1. À l'exception du texte d'André Demarchi, ce dossier présente des travaux discutés lors d'une journée d'étude tenue au Laboratoire d'ethnologie et de sociologie comparative à l'université Paris Nanterre, le 11 décembre 2015, dans le cadre du projet ANR Fabriq'Am «La fabrique des "patrimoines". Mémoires, savoirs et politiques en Amérique indienne aujourd'hui » (ANR-12-CULT-005). Nous remercions particulièrement Valentina Vapnarsky, Anath Ariel de Vidas et Philippe Erikson pour leur soutien dans l'organisation de cet événement et de ce dossier thématique.

2. Edmondson (2003, p. 14), à propos du Venezuela.

3. Rahier 1998; Pequeño 2004; Moreno 2007; Ramírez 2007; Stanfield 2013, p. 220-230; Sierra Becerra 2017. Ces auteurs montrent que depuis la fin des années 1980 l'inclusion dans les concours de Miss nationaux de candidates dont la couleur de peau n'est pas blanche a posé un dilemme parmi les élites urbaines : celui de la construction d'une identité nationale unifiée et singulière qui tienne compte des différences. Cela n'est évidemment pas restreint à l'Amérique latine. À partir d'une ethnographie de plusieurs concours de Miss America aux États-Unis, Sarah Banet-Weiser voit dans les « classic liberal stories » - la réalisation individuelle, le pluralisme et la tolérance - répétées pendant les concours et dans les médias conventionnels, une tentative de résoudre le problème d'une représentation unifiée de l'identité nationale (1999, p. 208). 
années 1920, à travers le cinéma d'Hollywood, les journaux d'actualités, puis les différents concours américains auxquels ont été invités à prendre part les élites nationalistes des grands centres urbains latino-américains ${ }^{4}$. C'est toutefois dans le cadre des festivités du calendrier catholique, plutôt que dans une fête nationale, que l'explorateur Laurent Saint-Cricq (alias Paul Marcoy) fournit, non sans humour, l'une des premières descriptions des pérégrinations d'une « reine amérindienne », depuis la mission franciscaine de Sarayacu dans l'Amazonie péruvienne du $\mathrm{XIX}^{\mathrm{e}}$ siècle:

La cérémonie ne commença qu'à neuf heures du soir. Au branle de la cloche, une femme désignée pour remplir les fonctions de reine de Noël entra dans l'église accompagnée de deux filles d'honneur, et alla s'agenouiller devant la balustrade du sanctuaire où l'attendait le révérend Plaza, entouré de vieux néophytes habillés en enfants de chœur et portant la croix et la bannière. La reine de Noël avait le visage bariolé de noir et de rouge. Un diadème de plumes de perroquet ornait son chef surmonté d'un immense peigne d'écaille. Des mouchoirs de cotonnade aux vives nuances, disposés en écharpe, rehaussaient la simplicité de son costume habituel. [...] Après que la reine, toujours agenouillée, eut satisfait aux quatre premières questions du catéchisme qui lui furent adressées en quechua par le prieur, celui-ci lui remit une petite corbeille matelassée dans laquelle était couché un EnfantJésus qu'elle embrassa dévotement; alors, se relevant et portant à deux mains son léger fardeau, Sa Majesté sortit de l'église, et, suivie de ses porte mèches, alla de maison en maison présenter le nouveau-né à l'adoration des fidèles. [...] La reine christophore mit plus d'une heure à faire sa tournée. Quand elle reparut au seuil de l'église, sa démarche était titubante, son peigne de travers et ses yeux hébétés. Ses filles d'honneur, vierges folles, avaient répandu l'huile de leur lampion, dont les mèches s'étaient éteintes. À mes questions sur l'état dans lequel se trouvaient la reine et ses suivantes, on répondit qu'il était d'usage d'offrir à Sa Majesté, au seuil de chaque maison où elle s'arrêtait avec l'Enfant-Jésus, un verre d'eau-de-vie dont elle buvait quelques gouttes. Si l'on se rappelle que Sarayacu compte cent soixante-six maisons, et qu'on admette par maison une moyenne de vingt gouttes, on s'étonnera, comme je m'étonnai, qu'après avoir ingurgité chacune trois mille trois cent vingt gouttes d'eau-de-vie, la reine et ses filles d'honneur pussent se tenir encore sur leurs jambes. (Marcoy 1869, p. 67-68)

4. Remarquons que si cette hypothèse nord-américaine permet sans doute d'expliquer l'homogénéité actuelle des mises en scène des concours nationaux, elle ne nous paraît pas suffisante pour comprendre l'émergence des concours de reine de beauté tout au long du $\mathrm{XX}^{\mathrm{e}}$ siècle dans les villages des zones rurales peu connectées avec les médias nationaux et les structures politiques des grandes villes. La prise en compte des multiples festivités catholiques (patronales, paroissiales, decembrinas, etc.) et autres fêtes populaires où la dévotion mariale peut donner lieu à la désignation d'une « marraine » ou d'une « reine des fêtes » pourrait éclairer l'adoption rapide de ces concours dans les festivités des populations vivant à proximité des territoires amérindiens. Sur l'air de famille entre représentation de la Vierge et Miss, voir Celigueta dans ce dossier et López García (2015, p. 178, 207). 
Dans ce dossier, c'est d'un autre genre de reines dont il est question. Celles-ci ne sont pas directement issues des festivités de la chrétienté coloniale, mais d'instances liées aux administrations territoriales des État-nations, dans le cas des concours officiels, ou à des organisations émanant de populations dites minoritaires pour les concours alternatifs. Dans les deux cas, les concours de Miss sont un spectacle parmi d'autres, emprunté à un large répertoire de performances - fanfares, parades, activités carnavalesques, etc. - souvent qualifié de « civil », pour le distinguer du répertoire cérémoniel ecclésiastique (messes, litanies, processions, etc.), bien que civil et religieux cohabitent ou fusionnent très régulièrement dans les célébrations populaires. L'expansion rapide des concours de beauté dans les régions les plus reculées de l'Amérique latine et la pluralité des façons dont les différents groupes sociaux, parmi lesquels des populations amérindiennes, se les approprient nous ont incité à nous pencher sur ces événements avec plus d'attention. Bien que tous les articles de ce dossier aient pour point de départ l'ethnographie d'un concours de Miss, chaque auteur a développé une problématique originale - historique, symbolique ou interactionniste. Cette introduction présente le dossier à partir d'une série de thèmes - ethnicité, représentation, patrimonialisation, beauté - qui traversent l'ensemble des textes. La diversité des références abordées vise à suggérer des pistes de réflexion et à provoquer des résonances au sein de cet objet de recherche peu exploré au carrefour du spectacle, de la politique, de l'esthétique et du genre. En effet, les articles composant ce dossier montrent que, dans les concours de Miss, la célébration de la beauté met en jeu une maîtrise de la représentation de l'identité collective et une production d'identités sexuées, tout en articulant certaines oppositions paradigmatiques des organisations sociales impliquées, tels que les pôles masculin et féminin, la tradition et la modernité, l'indigène et le Blanc.

\section{La Miss indigène, avatar glamour du multiculturalisme}

Dans le contexte latino-américain contemporain où les idéologies du métissage et les politiques assimilationnistes ont cédé la place - au moins officiellement à une gestion de la diversité en termes de « droit à la différence », et par-là à la reconnaissance d'identités minoritaires avec lesquelles les relations sont reformulées selon le principe du multiculturalisme ou de l'interculturalité, les concours de beauté apparaissent comme un des lieux possibles de négociation des relations entre les différents groupes ${ }^{5}$. Remarquons tout de suite que cet aspect se concrétise le plus souvent sur les podiums à travers la figure stéréotypée de « l'Indienne » qu'incarnent pendant un court moment les candidates blanches ou métisses lors des concours officiels. De fait, si « l'Indienne » apparaît dans 
beaucoup de concours de Miss, la participation « en chair et os » de candidates s'auto-identifiant indigènes ou appartenant à un groupe autochtone particulier est, elle, plus rare dès qu'il s'agit de concours mettant en jeu la représentation officielle d'une ville, d'une région ou d'une nation. Dans ces concours officiels, c'est lors de l'épreuve dite du costume « traditionnel », « typique » ou « folklorique » que des candidates issues de la société nationale s'ornent de coiffes de plumes, de colliers de graines, de jupes de fibres tissées et d'étoles de fourrure plus ou moins inspirés de traditions ornementales amérindiennes (Dziubinska dans ce dossier pour les métis d'Amazonie péruvienne ; voir aussi Hendrickson 1991). Il n'échappe à personne que les vêtements portés pendant cette épreuve dite du « costume typique » sont " sexier than the "real" thing " (Gustafson 2006, p. 365). L'un des effets recherchés serait de figurer l'union entre un modèle de corps désirable - celui de la jeune candidate blanche ou métisse - et un érotisme associé à la prétendue lasciveté ou disponibilité sexuelle des «Indiennes » (Canessa 2008, p. 44-45). Cette incorporation d'éléments amérindiens stylisés dans les concours officiels sous contrôle de la société dominante nous apparaît aussi, à la suite de Rogers (1999), comme une des multiples façons de marginaliser l'identité politique des groupes dits minoritaires en leur refusant un accès réel à la représentation officielle de la communauté politique.

Les contributions de ce dossier montrent que l'exclusion des concours de beauté officiels et la folklorisation de leurs traditions ornementales et chorégraphiques ne laissent pas toujours indifférents les Amérindiens et les mouvements ethniques. En Équateur le principal instigateur du concours Ñusta Andina, qui se revendique premier concours ethnique à l'échelle du pays, déclarait ainsi en 2015 à la presse nationale: «Les femmes de notre culture ont une beauté physique, de l'intelligence, une posture... mais lors des élections de reines ils nous mettaient des obstacles déguisés en critères requis et ils ne nous prenaient pas en compte » (El Comercio, 19 mars 2015). L'organisation ou la participation à des concours de Miss alternatifs apparaît comme une possibilité de contourner l'exclusion de la représentation officielle et de disputer le monopole de la définition de la beauté féminine aux organisateurs des concours conventionnels. Dans les concours maya analysés par Gemma Celigueta dans ce dossier, les podiums servent un militantisme ethnique qui s'oppose à la persistance de stéréotypes racistes parmi les populations formant la société nationale. L'auteure décrit les différents moments de la conquête par les Maya de Quetzaltenango d'un concours de Miss autrefois appelé India Bonita, alors entre les mains de l'élite ladino du Guatemala. Elle montre que la maîtrise des concours de Miss Maya, une affaire qui pourrait apparaître de l'extérieur comme frivole, met en jeu des luttes historiques ayant contribué à la formation d'identités collectives et à la légitimation de ces identités par la réélaboration d'une tradition esthétique, en même temps que l'accès des Maya aux instances représentatives de la ville. Avec la mise en place de concours de Miss, c'est en effet souvent la maîtrise 
des deux sens - politique et symbolique - de la notion de représentation qui est en jeu, à tel point qu'à Quetzaltenango les concours ont servi d'initiation aux mécanismes de la politique électorale pour une partie des Maya de la région.

Dans un dossier où la différence est très souvent exprimée dans les seuls termes de l'ethnicité, la contribution de Pascale Absi sur les concours de Miss Trans dans la Bolivie plurinationale est la bienvenue. En effet, à l'instar des concours indigènes, l'auteure montre que les concours transsexuels sont des lieux de revendication d'une inclusion d'autres modèles de femme et de féminité dans les espaces publics du pays. Monter sur le podium est une étape centrale dans la trajectoire qui conduit de la clandestinité à une identité de femme transsexuelle publiquement assumée. Le concours de beauté apparaît comme un dispositif de valorisation dans l'espace public d'une identité subjective ou collective différenciée; son but est d'atteindre une reconnaissance officielle de celle-ci.

Toutefois, comme le décrivent plusieurs auteurs de ce dossier, cette articulation entre des concours féminins et la revendication d'une identité singulière ne va pas sans poser un certain nombre de problèmes liés à l'ambivalence entre la reproduction de processus de subordination - en particulier pour les femmes -, la production d'une identité collective, régionale ou ethnique, et la possibilité d'une politique de représentation alternative dans l'agenda public des relations interethniques.

\section{La Miss, figure séduisante de médiation}

L'intégration de ce spectacle dans les festivités des Amérindiens signifie généralement l'adoption d'un régime de relations entre collectifs où la production d'images du corps politique et la mise en scène de son unité présumée à travers et au-delà de ses diverses composantes sont des éléments clés de la solidarité collective et des luttes de prestige. Dans les villages amérindiens des basses terres, comme ceux des Kakataibo d'Amazonie péruvienne analysés par Magda Dziubinska dans ce dossier, la mise en place de concours de beauté - phénomène récent - témoigne d'une intégration de ces communautés dans un système régional plus ample où le mode de relation officiel entre villages de même stature administrative passe en grande partie par un répertoire de festivités civiles souvent associées à la célébration de la nation. Dans ce cadre-là, une fois par an, les concours de Miss ajoutent à la « représentation-mandat » de l'homme politique tirant sa légitimité des épreuves électorales, une « représentation-incarnation » du corps politique dans la figure féminine de la Miss ${ }^{6}$. À travers des concours de beauté exclusivement féminins, c'est un processus de division des fonctions honorifiques selon une valorisation hiérarchisée des modes de représentation qui se met en branle.

6. Nous devons les formules « représentation-mandat » et « représentation-incarnation» à la lecture des travaux de philosophie politique de Diehl, Sintomer et Hayat (2014). 
Celle qui porte le titre honorifique de Miss ou de reine a comme principale fonction d'incarner une effigie au service du prestige de son groupe social ou de son village, c'est-à-dire de représenter avec splendeur et sensualité le corps politique dans les espaces de sa vie publique et diplomatique. En cela, la pratique fréquente en Amérique latine consistant à désigner des marraines (madrinas, quelques fois appelées señoritas) lors des événements impliquant des organisations de la société civile (équipes de sport, syndicats, écoles, etc.), tout comme celle propre au Brésil consistant à choisir des muses (musas) et des reines de batterie (rainha de bateria) lors des carnavals relèvent de la même logique que celle des concours de Miss ${ }^{7}$. Les candidates des premiers concours de Miss Kayapó du Brésil central, analysés dans ce dossier par André Demarchi, reprennent d'ailleurs les codes de l'ornementation corporelle des jeunes filles qui occupent le rôle récemment créé de rainhas, sorte de dames d'honneur aux parures exubérantes qui accueillent dans les villages kayapó les autorités venant des villes voisines. En dotant de belles jeunes femmes célibataires d'une fonction honorifique de représentation du groupe, il s'agit de fournir à ce groupe une figure de médiation avec l'extérieur qui soit féminine, inclusive et particulièrement séduisante. Les jeunes Miss ou reines font office à la fois d'emblèmes du groupe et d'intermédiaires entre les organisateurs, les participants et les invités - c'est-à-dire généralement entre des hommes - dans les événements auxquels elles participent. Elles peuvent ainsi être placées en tête des cortèges et affublées d'une écharpe ornée des motifs héraldiques du groupe, accompagner les hommes politiques lors de la réception de visiteurs de marque ou encore figurer en premier plan dans l'iconographie officielle. Alors que dans sa relation avec l'extérieur l'homme politique a pour fonction d'entraîner les autres par ses discours, la Miss doit mettre en scène son propre corps afin de susciter admiration et sympathie. Dans son article sur les concours kakataibo en Amazonie péruvienne, Dziubinska va plus loin: les concours de beauté seraient une excellente occasion de présenter aux alliés, métis et indigènes, les futures candidates au mariage et d'attirer des hommes étrangers dans la communauté.

À cet égard, la priorité donnée aux ornementations corporelles des femmes dans les espaces publics représente pour beaucoup de sociétés amérindiennes, en particulier dans les basses terres, une rupture par rapport aux pratiques

7. Il est aussi possible de situer les concours de Miss dans la continuité de célébrations inscrivant les femmes dans un parcours de vie distinct de celui des hommes. Une des cérémonies familiales parmi les plus importantes dans les sociétés nationales latinoaméricaines, qui a fait l'objet d'une appropriation dans certains contextes autochtones, est la fête d'anniversaire des quinze ans (quinceañera, ou festa de debutante au Brésil), dont il n'existe pas d'équivalent masculin. À la charge des parents et de leurs alliés, ce rite de puberté célèbre sous la forme d'un « mariage sans fiancé » (Napolitano 1997) l'entrée dans la maturité sexuelle des jeunes filles qui ont atteint leurs quinze ans. 
traditionnelles où ce n'est pas tant la beauté féminine qui s'expose publiquement que celle des hommes. Le foisonnement des parures et le chatoiement des couleurs peuvent alors être aussi bien un moyen d'impressionner de potentiels ennemis que d'indiquer son appartenance à une fonction ou un statut social ${ }^{8}$. On peut considérer l'apparition des concours de beauté parmi les populations amérindiennes comme l'indice de changements dans les manières de structurer les espaces publics avec l'instauration d'un champ de relations polarisé autour d'une dualité sexuelle, plutôt que d'un autre axe de différenciation (par exemple celui du couple affinité/consanguinité).

\section{La Miss, figure de l'intégrité et de la continuité culturelle}

Dans ces spectacles où l'enjeu principal pour les candidates est d'exhiber des qualités et des savoir-faire distinctifs considérés comme autant d'attributs d'une féminité idéale, la question des aptitudes déployées sur scène et celle des principes gouvernant la production des costumes, ornements et chorégraphies ne tardent pas à se poser. Ces questions apparaissent d'autant plus importantes que les différentes épreuves mettent en jeu des qualités et des valeurs souvent contradictoires. Typiquement, alors que pendant l'épreuve du « costume traditionnel » les candidates amérindiennes doivent faire la preuve d'une continuité avec des traditions chorégraphiques et ornementales locales, lors de l'épreuve du costume « de gala » ou « de soirée », c'est l'appropriation d'une esthétique urbaine, innovante et affriolante qui est valorisée. Dans la ville de Quetzaltenango, la question de l'adéquation des candidates et des reines à des canons esthétiques que les organisateurs imaginent comme ancestraux, notamment pour faire face au péril de l'acculturation, a nourri la plupart des conflits entre les groupes en charge du concours et se situe à l'origine des changements de règlement, d'épreuve et d'organisation. La classification par l'État guatémaltèque du concours de Quetzaltenango comme patrimoine culturel intangible de la Nation reflète certainement le poids accordé à la question de l'authenticité dans ce spectacle (Celigueta). On retrouve le même souci de présenter les performances des candidates comme une expression de la tradition chez les Kayapó du Brésil central. Dans cette société où l'ornementation corporelle constitue un patrimoine jalousement gardé, notamment parce que parures et peintures peuvent faire office d'emblèmes d'affiliation à des catégories sociales - dont la classe d'âge et le sexe -, le contrôle de la mise en scène du concours de Miss et des photos qui en sont prises sont de fait une

8. Nous renvoyons le lecteur à la littérature amazoniste qui a fourni de lumineuses analyses sur les modes d'apparence: Seeger (1975), Vidal (1981), Howard (1991), Turner (1995), Mentore (1993), Gallois (2002), Erikson (2003), Taylor (2003), Franchetto (2003), Alès (2010), Lagrou (2011) et Hugh-Jones (2014). 
priorité (Demarchi). Mais face à des membres du jury qui ignorent ce qu'il est acceptable de faire, selon les Kayapó, sur le tapis rouge du concours ou avec les photographies des candidates, ces derniers ont exigé auprès des organisateurs (une municipalité brésilienne voisine) un plus grand contrôle de l'événement, allant jusqu'à l'interdire. L'article de Dziubinska montre que les Kakataibo font preuve d'une plus grande souplesse dans l'épreuve du « costume typique ». La reprise des anciennes coutumes d'ornementation corporelle impliquerait de voir défiler les adolescentes à moitié nues. Cela provoquerait une stigmatisation en termes de « sauvagerie », stigmatisation dont ce groupe essaie de se défaire et qui serait peu compatible avec l'ambition générale de ces concours, où il s'agit moins d'affirmer la maîtrise d'une tradition ethnique distinctive que celle d'un glamour moderne et mondain. Les candidates puisent alors dans un imaginaire esthétique aujourd'hui partagé par les métis et les Kakataibo, fruit des rapports interethniques dans la région.

Bien que souvent organisés au nom d'une revitalisation culturelle ou d'une discrimination positive, les concours ethniques et genrés sont fréquemment en rupture avec les esthétiques propres aux groupes qui les organisent en copiant celles des groupes socialement dominants. Au Brésil, face aux polémiques engendrées par le concours de Miss Kayapó, la FunAI (Fundação Nacional do Índio, agence étatique en charge des affaires indigènes) a produit un communiqué qui résume bien les critiques que suscite la tenue de concours de beauté indigène : « [...]. De telles initiatives ne contribuent pas à la promotion de la diversité sociale brésilienne, mais renforcent plutôt des vues partiales sur les femmes, déconstruisent les savoirs autochtones et dévaluent la valeur symbolique des femmes dans les différentes sociétés autochtones $\gg{ }^{9}$. Certains commentateurs des concours maya de Quetzaltenango ajoutent une dimension militante: en se focalisant sur des revendications d'ordre esthétique, les concours de reines de beauté détourneraient les populations des luttes plus fondamentalement politiques (Celigueta).

\section{La Miss, figure de transformations}

On peut penser que la représentation symbolique du corps politique produite par les concours de Miss vis-à-vis de l'extérieur participe à la transformation des attributs féminins au sein des sociétés amérindiennes. L'établissement d'un lien entre le destin d'un groupe dans sa totalité - hommes et femmes confondus - et une personne spécialement distinguée pour sa féminité est peut-être un des éléments les plus signifiants de cette transformation. Ce lien ne se constitue pas seulement à travers les défilés en costumes, mais pendant une épreuve où les candidates sont amenées à témoigner, par un bref discours,

9. Communiqué officiel de la FunAI : http://www.funai.gov.br/index.php/comunicacao/ notas/2791-nota-funai-miss-brasil-indigena, consulté le 01/03/17. 
de leur engagement à défendre les intérêts du groupe et à faire la promotion d'une vie en communauté respectueuse des valeurs les plus consensuelles. Par-là, les concours apparaissent comme un premier pas dans la formation d'un leadership féminin ${ }^{10}$. «Tout a commencé quand j'ai été élue reine lors du concours de Miss indigène à Pucallpa », affirmait ainsi Diana Mori à Magda Dziubinska, pour expliquer son parcours de militante shipibo engagée dans plusieurs projets de défense des droits territoriaux des peuples indigènes au Pérou. Encore une fois, cet effet des concours n'est pas propre à ceux définis en termes ethniques: « le fait d'avoir gagné des concours de beauté m'a énormément aidé $[\ldots]$ à ce que je me définisse comme femme transsexuelle et que je devienne une militante » confie une des premières Miss Bolivie Transexuelle à Pascale Absi dans ce dossier.

Au-delà de ces effets de subjectivation en vue d'un leadership se déployant dans les espaces intermédiaires entre le groupe et l'extérieur, la question de la promotion par les concours d'une esthétique hors du commun est également abordée par les auteurs de ce dossier. Chacun décrit à la fois les conceptions locales de la beauté et la manière dont les différents régimes du beau, souvent contradictoires, s'intriquent dans les concours de Miss indigène. En mettant en lumière les continuités dans les manières de percevoir le beau et d'embellir les corps féminins, Demarchi insiste sur les nombreuses analogies entre les concours de Miss et les rituels de nomination destinés aux jeunes filles. Il est vrai que les concours de Miss Kayapó ne ressemblent à aucun autre par l'ampleur du traitement corporel des impétrantes et qu'il existe déjà dans ce groupe un important appareil initiatique dédié à la polarisation homme-femme. Les deux cérémonies sont réservées aux filles pubères qui incarnent ce qui est considéré comme la perfection dans l'esthétique kayapó - le corps féminin jeune, ferme et sexuellement désirable. L'enjeu explicite de ces deux pratiques est de rendre encore plus beau le corps des filles, plus séduisant et surtout immanquablement féminin. À la différence de cette continuité que Demarchi a pu observer au Brésil central, Magda Dziubinska montre comment l'école et les concours de beauté organisés par les professeurs métis introduisent un nouveau canon de beauté parmi les adolescents kakataibo d'Amazonie péruvienne ${ }^{11}$. La minceur, qui en fait partie, est de plus en plus valorisée et recherchée par les jeunes femmes, alors que ce sont des corps féminins forts et robustes que préfèrent les Kakataibo plus âgés, la maigreur étant généralement associée à la maladie, au manque de force

10. Le passage du concours de beauté aux compétitions électorales semble régulier chez les populations autochtones d'Amérique du Nord (Bousquet et Morissette 2014; Williams 2013; Cohen et al. 1996) et, dans une certaine mesure, dans les concours maya (Celigueta dans ce dossier; McAllister 1996; Schackt 2005).

11. On retrouve là le rôle de l'institution scolaire en Amazonie dans la transmission des codes de célébration des États-nations, à travers l'enseignement des valeurs, hymnes et chorégraphies qui sont au cœur de ces cérémonies (voir Rival 1996; Collet 2006; Macedo 2009). 
et à l'altération des rapports sociaux. Bien que les concours de Miss kayapó et kakataibo soient différents, tant du point de vue de l'organisation que de celui de la manière dont ils mobilisent et manipulent l'imagerie de l'indianité, les deux concours éclairent le lien entre la beauté et l'imitation de la gestuelle des mannequins professionnels. Les contributions de ce dossier insistent ainsi sur le travail et les techniques du corps visant à incarner une certaine beauté féminine - le « glamour» des Miss. En plus de l'ornementation corporelle, candidater à un concours implique en effet l'incorporation d'une série de dispositions, parfois laissées implicites, contrastant avec les habitudes ordinaires : démarche, postures, regards, expressions faciales, port de tête, mouvements des mains, autant de marqueurs de différenciation entre les sexes d'une part, et d'autre part entre les « femmes du commun » et les Miss. On retrouve d'ailleurs dans plusieurs photographies accompagnant ce dossier le fameux «bashful knee bend » identifié par Goffman (1976, p. 45) dans les annonces publicitaires américaines: une jambe avancée par rapport à l'autre et le genou légèrement plié, de sorte à produire une posture courbée dans laquelle le mouvement est arrêté afin de s'offrir aux regards. Mais il ne s'agit pas seulement de défiler en se déhanchant, l'enjeu est d'associer les mouvements sensuels à une certaine disposition affective combinant la joie et l'audace, transformant ainsi la performance en un exercice de séduction. Dans les deux cas amazoniens, cette mise en mouvement particulière du corps constitue un des critères de beauté décisif pour gagner la compétition.

L'exemple le plus frappant de transformation corporelle présenté dans ce dossier est celui des femmes transsexuelles boliviennes décrit par Absi. Le principal enjeu de ces transformations est de produire un corps nouveau, exhibant une identité féminine, ce qui implique la prise d'hormones, des injections de silicone et souvent une réassignation génitale. Le concours de beauté offre ici un cadre idéal pour mettre en scène ces corps transformés et valider ainsi la transition. Une fois la ressemblance avec la femme « naturelle » établie, chaque candidate doit encore incorporer les codes esthétiques et comportementaux d'une femme glamour hypersexuée qui incarne le fantasme de ce que Deborah Elliston a désigné comme la " Global Femme »" C'est ainsi que selon Absi le collectif LGBT bolivien reproduit dans ses concours de Miss la féminité dans ce qu'elle a de plus exacerbé et stéréotypé. Remarquons au passage que ce modèle de féminité mondaine et glamour n'est pas complètement absent des villages amérindiens mais, comme le montrent les articles de Dziubinska et Demarchi, il y parvient de manière indirecte, médiatisée par les autres populations locales,

12. En résonance avec d'autres auteurs (notamment Asayag 1999, Alexeyeff 2000, Besnier 2002 et Balogun 2012), Elliston décrit ce modèle de féminité comme «a mass mediated Euro-American fashion-model feminity, beautiful in cover model ways - thin, ever-youthful, scantily clad, made up in cosmetics, glamorous, and white » (2014, p. 44). 
par exemple au sein de l'école ou, précisément, par le biais des concours de beauté. Dans la continuité de la pensée de Butler (1993), Absi suggère que la mise en scène de la femme "sur-produite » lors des concours de beauté transsexuels pourrait être perçue comme une stratégie adoptée par les femmes transsexuelles dont l'enjeu est de se faire accepter dans un premier temps en tant que femme, afin de subvertir ensuite cette catégorie en l'ouvrant à des expériences alternatives de la féminité.

Finalement, nous espérons que ce dossier thématique consacré aux concours de beauté témoigne, grâce à la diversité des cas étudiés et des perspectives adoptées, de la richesse et de l'intérêt de cet objet de recherche original, parfois déconcertant mais assurément flamboyant, où le politique s'entrelace avec l'esthétique dans un décorum gai et kitch. Si l'on en juge par l'enthousiasme manifesté tant par les intervenants à la journée d'étude que par le public nombreux venu les écouter, le pari d'aborder sérieusement les coulisses des concours de beauté a été gagné; s'il y a des reines dans les sociétés sans roi, il y a mystère.

\section{Références citées}

ALÈs Catherine

2010 «Art corporel, savoir et engendrement chez les Yanomami », in Gilles Boëtsch, Dominique Chevé et Hélène Claudot-Hawad (éd.), Décors des corps, CNRS Éditions, Paris, p. 331-341.

AleXeyefF Kalissa

2000 «Dragging drag: The performance of gender and sexuality in the Cook Islands », The Australian Journal of Anthropology, 11 (2), p. 97-307.

AsSAYAG Jackie

1999 «La "glocalisation” du beau. Miss Monde en Inde, $1996 »$, Terrain, 32, p. 67-82. BALOGun Oluwakemi

2012 «Cultural and cosmopolitan. Idealized femininity and embodied nationalism in Nigerian Beauty Pageants », Gender \& Society, 26 (3), p. 357-381.

BANET-Weiser Sarah

1999 The most beautiful girl in the world. Beauty pageants and national identity, University of California Press, Berkeley/Los Angeles/London.

BESNIER Niko

2002 « Transgenderism, locality, and the Miss Galaxy beauty pageant in Tonga », American Ethnologist, 29, p. 534-566.

Bousquet Marie-Pierre et Anny Morisette

2014 «Reines, princesses, Miss et majorettes. Une construction de la féminité chez les Amérindiens et Amérindiennes du Québec ( $\mathrm{xx}^{\mathrm{e}}-\mathrm{XXI}^{\mathrm{e}}$ siècles) », in Gilles Havard et Frédérick Laugrand (éd.), Éros et tabou. Sexualité et genre chez les Amérindiens et les Inuit, Septentrion, Québec, p. 359-407. 
BORLAND Katherine

1996 «The India Bonita of Monimbó. The politics of ethnic identity in the New Nicaragua », in Collen Ballerino Cohen, Richard Wilk et Beverly Stoeltje (éd.), Beauty queens on the global stage, Routledge, New York/London, p. 75-88.

ButLER Judith

1993 Bodies that matter. On the discursive limits of « sex », Routledge, Londres/ New York.

CANESSa Andrew

2008 «Sex and the citizen. Barbies and beauty queens in the age of Evo Morales », Journal of Latin American Cultural Studies, 17 (1), p. 41-64.

Cohen Ballerino Colleen, Richard R. WiLk et Beverly StoeltJe

1996 Beauty queens on the global stage: gender, contests, and power, Routledge, New York.

Collet Celia

2006 Ritos de civilizaçao e cultura: a escola bakairi, thèse de doctorat, département d'Anthropologie sociale, Universidade Federal do Rio de Janeiro.

Dienl Paula, Yves Sintomer et Samuel Hayat

2014 «Introduction au dossier. La représentation politique », Trivium [en ligne], 16, http://trivium.revues.org/4771, consulté le 28/04/17.

EDMONDSON Belinda

2003 «Public spectacles. Caribbean women and the politics of public performance », Small Axe, 7 (13), p. 1-16.

ELLISTON Deborah

2014 «Queer history and its discontents at Tahiti: the contested politics of modernity and sexual subjectivity », in Niko Besnier et Kalissa Alexeyeff (éd.), Gender on the edge. Transgender, gay, and other Pacific islanders, University of Hawai'i Press, Honolulu, p. 33-55

ERIKSON Philippe

2003 " "Comme à toi jadis on l'a fait, fais-le moi à présent..." Cycle de vie et ornementation corporelle chez les Matis (Amazonas, Brésil) », L'Homme, 167-168 (3-4), p. 129-152.

FranchetTo Bruna (éd.)

2003 Ikú ügühütu higei. Arte gráfica dos povos karib do alto Xingu, Museo do Indio/FUNAI, Rio de Janeiro.

Gallois Dominique

2002 Kusiwa: pintura corporal e arte gráfica waiãpi, Museu do Índio/FUNAI/CTI/ NHI/USP, Rio de Janeiro.

GoFFMAN Erving

1976 Gender advertisements, Harper Torchbooks, New York/Cambridge/San Francisco. GuSTAFSON Bret

2006 «Spectacles of autonomy and crisis. Or, what bulls and beauty queens have to do with regionalism in Eastern Bolivia », Journal of Latin American Anthropology, 11 (2), p. 351-379. 
Les concours de beauté au prisme des différences en Amérique latine

\section{HENDRICKSON Carol}

1991 «Images of the Indian in Guatemala: the role of Indigenous dress in Indian and Ladino constructions ", in Greg Urban et Joel Sherzer (éd.), Nation-States and Indians in Latin America, University of Texas Press, Austin, p. 287-306.

HowARD Catherine

1991 «Fragments of the heavens: feathers as ornaments among the Waiwai », in Ruben E. Reina et Kenneth M. Kensinger (éd.), The gift of birds: featherworking of Native South American Peoples, University Museum-University of Pennsylvania, Philadelphia, p. 50-69.

Hugh-JONEs Stephen

2014 «Caixa de Pandora: estilo alto-rio-negrino », Revista de antropologia da UFSCar, 6, p. 155-173.

LAGrou Elsje

2011 «Le graphisme sur les corps amérindiens. Des chimères abstraites?», Gradhiva. Revue d'anthropologie et d'histoire des arts, 13, p. 69-93.

LÓPEZ García Julían

2015 «Reinas indígenas de Guatemala en el siglo xxI: melancolía, orgullo y coraje », in Manuel Gutiérrez Estévez et Alexandre Surrallés (éd.), Retórica de los sentimientos: etnografias amerindias, Iberoamericana-Vervuert, Madrid, p. 177-231.

MACEDO Silvia

2009 «Indigenous school policies and politics. The sociopolitical relationship of Wayapi Amerindians to Brazilian and French Guianan schooling », Anthropology and Education Quarterly, 40, p. 170-186.

MarCOY Paul

1869 Voyage à travers l'Amérique du Sud, de l'Océan Pacifique à l'Océan Atlantique, Hachette, Paris.

McAllister Carlota

1996 « Authenticity and Guatemala's Maya Queen », in Collen Ballerino Cohen, Richard Wilk et Beverly Stoeltje (éd.), Beauty queens on the global stage, Routledge, New York/London, p. 105-124.

Mentore George

1993 « Tempering the social self: body adornment, vital substance, and knowledge among the Waiwai », Journal of Archeology and Anthropology, 9, p. 22-23.

Moreno María

2007 «Misses y concursos de belleza indígena en la construcción de la nación ecuatoriana », Íconos. Revista de Ciencias Sociales, 28, p. 81-91.

NAPOLITANO Valentina

1997 «Becoming a Mujercita: rituals, fiestas and religious discourses », The Journal of the Royal Anthropological Institute, 3 (2), p. 279-296.

PequeÑo Andrea

2004 «Historias de misses, historias de naciones », Íconos. Revista de Ciencias Sociales, 20, p. 114-117.

RAHIER Jean

1998 « Blackness, the racial/spatial order, migrations, and Miss Ecuador 1995-96 », American Anthropologist, 100 (2), p. 421-430. 
RAMíREz Ingrid J.

2007 «Reinados de belleza y nacionalización de las sociedades latinoamericanas », Íconos. Revista de Ciencias Sociales, 28, p. 71-80.

RIVAL Laura

1996 «Formal schooling and the production of modern citizens in the Ecuadorian Amazon », in Bradley A. Levinson, Douglas E. Foley et Dorothy C. Holland (éd.), The cultural production of the educated person, State University of New York Press Albany, p. 153-168.

ROGERS Mark

1999 « Spectacular bodies: folklorization and the politics of identity in ecuadorian beauty pageants », Journal of Latin American Anthropology, 3 (2), p. 54-85.

SCHACKT Jon

2005 «Mayahood through beauty: Indian beauty pageants in Guatemala », Bulletin of Latin American Research, 24 (3), p. 269-287.

SeEger Anthony

1975 « The meaning of body ornaments: a Suya example », Ethnology, 14 (3), p. 211-224.

Sierra BeCERra Diana C.

2017 « The first black Miss Colombia and the limits of multiculturalism », Latin American and Caribbean Ethnic Studies, 12 (1), p. 71-90.

StAnField Michael E.

2013 Of beasts and beauty: gender, race, and identity in Colombia, University of Texas Press, Austin.

TAYLOR Anne-Christine

2003 «Les masques de la mémoire. Essai sur la fonction des peintures corporelles jivaro », L'Homme, 165 (1), p. 223-248.

TURNER Terence

1995 « Social body and embodied subject: bodiliness, subjectivity and sociality among the Kayapó », Cultural Anthropology, 10 (2), p. 143-179.

VIDAL Lux

1981 «Contribution to the concept of person and self in Lowland South America: body painting among the Kayapo-Xikrin », in Contribuçoes à antropologia em homengaem ao Professor Egon Schaden, Museu Paulista, Saõ Paulo, p. 291-302.

Williams Caroline

2013 It's not a beauty pageant! An examination of leadership development through Alaska native pageants, University of Arizona, Tucson.

WroblewsKi Michael

2014 «Public Indigeneity, Language Revitalization, and Intercultural Planning in a Native Amazonian Beauty Pageant », American Anthropologist, 116 (1), p. 65-80. 